Research Article

\title{
Increased Levels of ER Stress and Apoptosis in a Sheep Model for Pulmonary Fibrosis Are Alleviated by In Vivo Blockade of the KCa3.1 Ion Channel
}

\author{
Udari E. Perera $\left(\mathbb{D},{ }^{1}\right.$ Louise Organ $\left(\mathbb{D},{ }^{2}\right.$ Sasika N. V. Dewage $\mathbb{D}^{1},{ }^{1}$ Habtamu B. Derseh $\left(\mathbb{D},{ }^{1}\right.$ \\ Andrew Stent $₫{ }^{1}{ }^{1}$ and Kenneth J. Snibson ${ }^{1}$ \\ ${ }^{1}$ School of Veterinary Science, The University of Melbourne, Parkville, VIC, Australia \\ ${ }^{2}$ Nottingham Respiratory Research Unit, University of Nottingham, Nottingham, UK \\ Correspondence should be addressed to Udari E. Perera; udari.perera@unimelb.edu.au
}

Received 16 November 2020; Revised 4 March 2021; Accepted 10 March 2021; Published 20 March 2021

Academic Editor: Pierachille Santus

Copyright ( $\odot 2021$ Udari E. Perera et al. This is an open access article distributed under the Creative Commons Attribution License, which permits unrestricted use, distribution, and reproduction in any medium, provided the original work is properly cited.

\begin{abstract}
Idiopathic pulmonary fibrosis (IPF) is a fatal interstitial lung disease, characterized by progressive damage to the lung tissues. Apoptosis and endoplasmic reticulum stress (ER stress) in type II alveolar epithelial cells (AECs) and lung macrophages have been linked with the development of IPF. Therefore, apoptosis- and ER stress-targeted therapies have drawn attention as potential avenues for treatment of IPF. The calcium-activated potassium ion channel KCa3.1 has been proposed as a potential therapeutic target for fibrotic diseases including IPF. While KCa3.1 is expressed in AECs and macrophages, its influence on ER stress and apoptosis during the disease process is unclear. We utilized a novel sheep model of pulmonary fibrosis to demonstrate that apoptosis and ER stress occur in type II AECs and macrophages in sheep with bleomycin-induced lung fibrosis. Apoptosis in type II AEC and macrophages was identified using the TUNEL method of tagging fragmented nuclear DNA, while ER stress was characterized by increased expression of GRP-78 ER chaperone proteins. We demonstrated that apoptosis and ER stress in type II AECs and macrophages increased significantly 2 weeks after the final bleomycin infusion and remained high for up to 7 weeks post-bleomycin injury. Senicapoc treatment significantly reduced the rates of ER stress in type II AECs and macrophages that were resident in bleomycin-infused lung segments. There were also significant reductions in the rates of apoptosis of type II AECs and macrophages in the lung segments of senicapoc-treated sheep. In vivo blockade of the KCa3.1 ion channel alleviates the ER stress and apoptosis in type II AECs and macrophages, and this effect potentially contributes to the anti-fibrotic effects of senicapoc.
\end{abstract}

\section{Introduction}

Idiopathic pulmonary fibrosis (IPF) is a fatal interstitial lung disease characterized by progressive damage to the lung tissues, which in turn leads to the impairment of the gas exchange function of the lungs [1-3]. While pirfenidone and nintedanib are two drugs currently approved by the FDA for slowing the progression of IPF [4-7], these drugs do not cure the ailment [4-7]. Investigation of potential therapeutic targets to inhibit the ongoing fibrosis remains a major challenge due to a lack of understanding of the pathogenesis of fibrosis in the lungs.

The progression of IPF involves many disease elements including activation of transforming growth factor $\beta$ (TGF$\beta$ ) and other chemokines, type II alveolar epithelial cell (AECs) apoptosis, ineffective removal of apoptotic cells, and aberrant wound healing and endoplasmic reticulum (ER) stress $[8,9]$. The ER is a special organelle normally responsible for the folding synthesized proteins and subsequent vesicular transport of proteins to the Golgi apparatus. 
Factors such as $\mathrm{Ca}^{2+}$ depletion, redox homeostatic alteration, nutrient deprivation, and environmental insults can affect these folding processes, eventually leading to accumulation of unfolded proteins which disrupt the ER function, resulting in ER stress [10].

ER stress is known to initiate apoptosis pathways [11] through activation of caspase-12. Caspase-12 enables specific cleavage of caspase-9, which together with downstream effects eventually leads to apoptosis [11].

ER stress in type II AECs is one of the prominent features of IPF and is frequently found in patients with familial and sporadic IPF [12]. ER stress has also been observed in macrophages in the lungs of patients with asbestosis [13]. ER stress induces inflammatory signaling, epithelial-mesenchymal transition, myofibroblast activation, macrophage polarization, and T cell differentiation that may contribute to the disease progression of IPF [14-17].

The calcium-activated potassium ion channel KCa3.1 has been proposed as a potential therapeutic target for fibrotic diseases including IPF [18-21]. The KCa3.1 ion channel is expressed in a number of cell types in the lungs and other tissues $[18,20,22]$. Importantly, stimulation of the KCa3.1 ion channel activates cells such as fibroblasts, macrophages, and epithelial cells that are involved in the disease processes of IPF [18]. We have recently reported that blockade of the KCa3.1 ion channel using senicapoc (ICA-17043) attenuates interstitial lung fibrosis, improves lung compliance, and attenuates microvascular remodeling in a sheep model for pulmonary fibrosis $[23,24]$. In the current study, we utilized the sheep model to analyze the regulatory function(s) of the KCa3.1 ion channel in type II AECs and macrophages in fibrotic disease.

Our aims in the present study are to firstly ascertain whether ER stress and increased rates of apoptosis in type II AECs and macrophages are observed in sheep lungs with experimentally induced fibrosis. Secondly, we investigate whether blockade of KCa3.1 ion channel with a specific inhibitor, senicapoc, will retard ER stress and the rate of apoptosis in type II AECs and macrophages in this large animal model of pulmonary fibrosis.

\section{Materials and Methods}

2.1. In Vivo Experimental Design to Evaluate ER Stress and Apoptosis in Type II AEC and Macrophages in BleomycinInduced Lung Fibrosis Using Sheep Model. All experimental procedures relating to sheep experiments and sample collection were approved by Animal Experimentation Ethics Committee, University of Melbourne (Parkville, VIC, Australia) and adhere to the Australian Code of Conduct for the Care and Use of Animals for Scientific Purposes. Sheep lung tissue samples were obtained from another study [25] and the experimental design was briefly as follows.

Pulmonary fibrosis was induced in eight female merino sheep $(n=8)$ aged within 9-12 months, using Bleomycin sulphate (Hospira, Melbourne, Victoria, Australia) prepared at a concentration of $0.6 \mathrm{U}$ bleomycin $/ \mathrm{ml}$ saline [25]. From the prepared bleomycin solution, $5 \mathrm{ml}$ was administered as bolus into the desired lung segment via the biopsy port of the fiber-optic bronchoscope (total dose of $3 \mathrm{U}$ bleomycin/lung segment). Similarly, $5 \mathrm{ml}$ of $0.9 \%$ sterile saline was administered to the contralateral caudal lung segment as an internal control (Figure 1). The second dose of bleomycin/ saline was administered to the same lung segments after 14 days (Figures 1(a) and 1(b)). Then, the sheep were kept another 14 days to develop lung fibrosis. All the sheep were sacrificed at day 28 by administering intravenous barbiturate (Lethabarb, Virbac Animal Health, Australia).

2.2. Necropsy and Tissue Sampling. Lungs were removed during the necropsy and the targeted lung segments were identified. Caudal segments of the targeted lung lobes were separated out and cannulated by exposing the bronchiole to inflate the segments with a $1: 1$ mixture of optimal cutting temperature (OCT) and sterile PBS solution under pressure of approximately $20 \mathrm{~cm} / \mathrm{H}_{2} \mathrm{O}$, in order to preserve the lung tissue architecture during processing. Then, $0.5 \mathrm{~cm}$ thickness lung tissue samples were fixed in $4 \%$ paraformaldehyde followed by $70 \%$ ethanol and processed in paraffin for histopathological analysis.

\subsection{Evaluation of ER Stress in Type II AEC and Macrophages.} Persistent injury to type II AECs and lung macrophages potentially induces ER stress, which in turn can progress to apoptosis. Glucose regulated protein (GRP) 78 is one of the best-characterized ER chaperone proteins and serves as a classic marker to evaluate ER stress. Immunohistochemistry was performed using a rabbit monoclonal anti-GRP78 primary antibody (Abcam (ab108615)) to evaluate ER stress in type II AECs and macrophages on paraffin lung sections. Each assay was performed with a negative control without adding the primary antibody.

\subsection{Anti-GRP78 Immunohistochemical Staining. Paraffin} sections were subjected to deparaffinization and rehydration by immersing them in xylene for three $5 \mathrm{~min}$ changes, followed by two $5 \mathrm{~min}$ changes of absolute ethanol, followed by $70 \%$ ethanol for $5 \mathrm{~min}$. Antigen retrieval was performed using pre-heated citrate buffer ( $\mathrm{pH}-6)$ heated for $15 \mathrm{~min}$, and the slides were left to cool down for $10 \mathrm{~min}$ followed by PBS washing. Then, the slides were kept in $3 \% \mathrm{H}_{2} \mathrm{O}_{2}$ for 10 min to block endogenous peroxides and rinsed thoroughly with PBS. Undiluted fetal calf serum (FCS) was added to each slide and incubated for 1 hour to block non-specific bindings with the antigen. Anti-GRP78 antibody (Abcam, ab108615) diluted 1:50 with FCS was added to each sample and incubated for 1 hour. Slides were rinsed gently with PBS and then EnVision ${ }^{\mathrm{TM}}$ dual link system-HRP (Horseradish Peroxidase) (Dako, North America Inc., CA, USA) was applied and incubated for $30 \mathrm{~min}$. To visualize the antigen-antibody reaction, Nova RED peroxidase substrate (Vector Laboratories Inc., CA, USA) was added to each sample and incubated for $3 \mathrm{~min}$. Then, the samples were washed with distilled water to stop the reaction and counter-stained with Hematoxylin. 


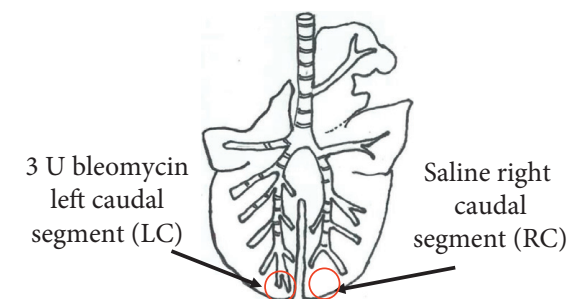

(a)

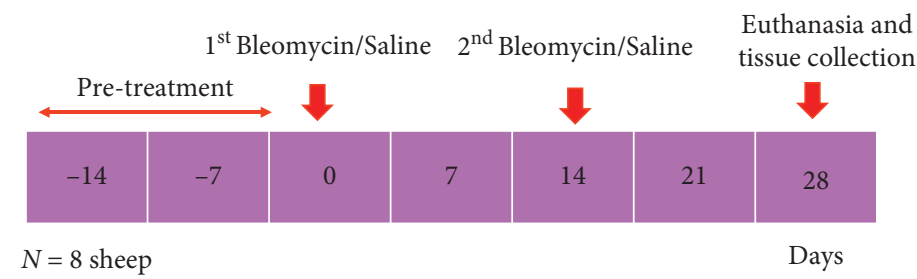

(b)

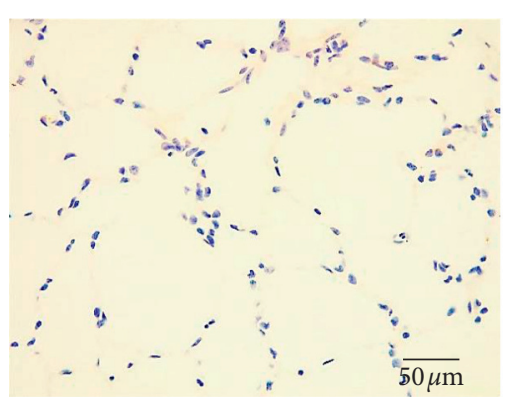

GRP78 +ve (Saline)

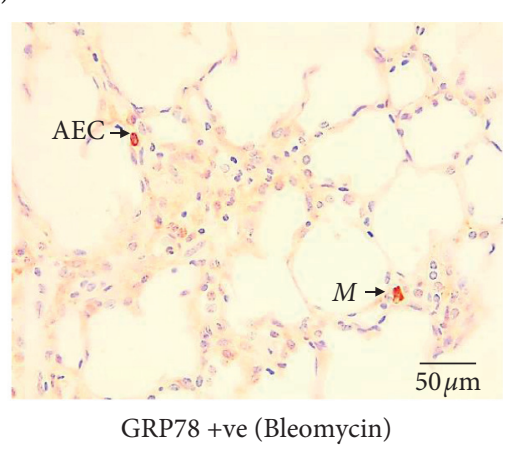

(c)

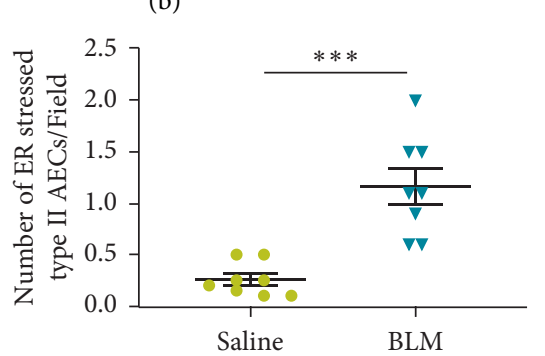

(d)

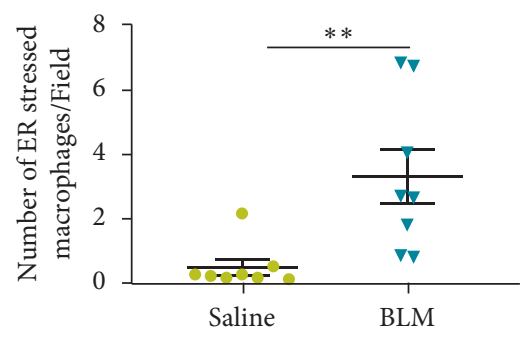

(e)

FIGURE 1: Schematic diagram of bleomycin infusion protocols in sheep $(n=8)$ and immunohistochemical staining against GRP78 to identify ER stressed type II AECs and macrophages in sheep parenchymal lung tissues. (a) In individual sheep $(n=8)$, the left caudal lung segment received infusions of bleomycin, while the right caudal lung segment received saline for internal control purposes. (b) Diagram shows the timeline for bleomycin/saline infusions and the tissue collections. These infusions were repeated at day 14 and tissue samples were harvested at day 28. All the animals were kept for 14 days acclimation period (pre-treatment) prior to bleomycin/saline administration. (c) Representative images of GRP78 immuno-stained sections taken from the differentially treated lung segments. Arrows indicate ER stressed type II alveolar epithelial cells (AECs) and macrophages (M). (d, e) Graphs represent the number of ER stressed type II AECs and macrophages in bleomycin- (BLM-) and saline-infused lung segments ( $n=8$ sheep). The data was taken from twenty representative, non-overlapping fields randomly captured under $40 \mathrm{x}$ magnification. Each bar represents the mean \pm standard error of the mean. Significance was determined by $t$ test. ${ }^{* *} p<0.001$ and ${ }^{* * *} p<0.0001$.

\subsection{Evaluation of Apoptosis in Type II AEC and Macrophages.} Click-iT ${ }^{\mathrm{TM}}$ Colorimetric Terminal Deoxynucleotidyl Transferase-dUTP Nick End Labeling (TUNEL) assay (Click-iT $^{\mathrm{TM}}$ TUNEL Colorimetric IHC Detection Kit, Thermo Fisher Scientific, USA) was performed on paraffin lung tissue sections to evaluate type II AECs and macrophages turnover in bleomycin-induced lung fibrosis in sheep model (Figure 2). Each assay was performed with a negative control without adding TdT reaction mixture. Paraffin sections were deparaffinized and rehydrated by xylene and a graded series of ethanol according to the manufacturer's instructions (Click-iT ${ }^{\mathrm{TM}}$ TUNEL Colorimetric IHC Detection Kit, Thermo Fisher Scientific, USA). The slides were dipped in $1 \% \mathrm{H}_{2} \mathrm{O}_{2}$ for $10 \mathrm{~min}$ to block endogenous peroxidase. Diluted Proteinase K (dilution rate $1: 50$ ) was added to each sample and incubated for $5 \mathrm{~min}$. Then, the samples were blocked with blocking buffer (1\% Bovine Serum Albumin $(\mathrm{BSA})+5 \%$ Normal Sheep Serum (NSS) + PBS) for $30 \mathrm{~min}$. Slides were washed thoroughly with PBS for $5 \mathrm{~min} 3$ times prior to each step. For the Terminal Deoxynucleotidyl Transferase (TdT) reaction, slides were incubated with TdT reaction buffer for $10 \mathrm{~min}$. TdT reaction mixture was prepared according to the manufacturer's protocol and added to each slide and incubated for $60 \mathrm{~min}$. After rinsing with PBS to fully quench the TdT reaction, slides were then immersed in Saline Sodium Citrate (SSC) buffer for $15 \mathrm{~min}$ and then in PBS and click-iT TUNEL Colorimetric washing solution. Click-iT TUNEL Colorimetric reaction cocktail (Click-iT reaction buffer $+\mathrm{CuSo} 4$, biotin azide + reaction buffer additive) was prepared according to the manufacturer's recommendation, added to each sample, and incubated for $30 \mathrm{~min}$. Samples were washed with PBS and Click 


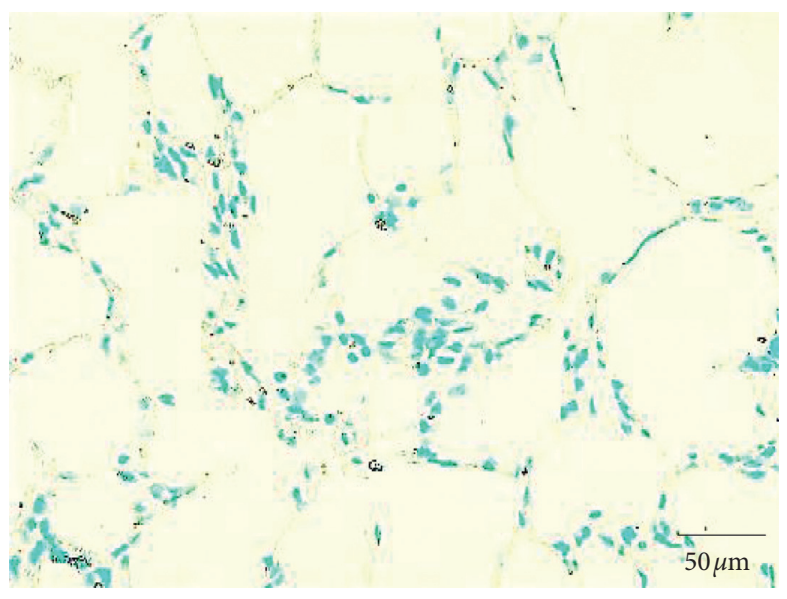

TUNEL +ve (Saline)

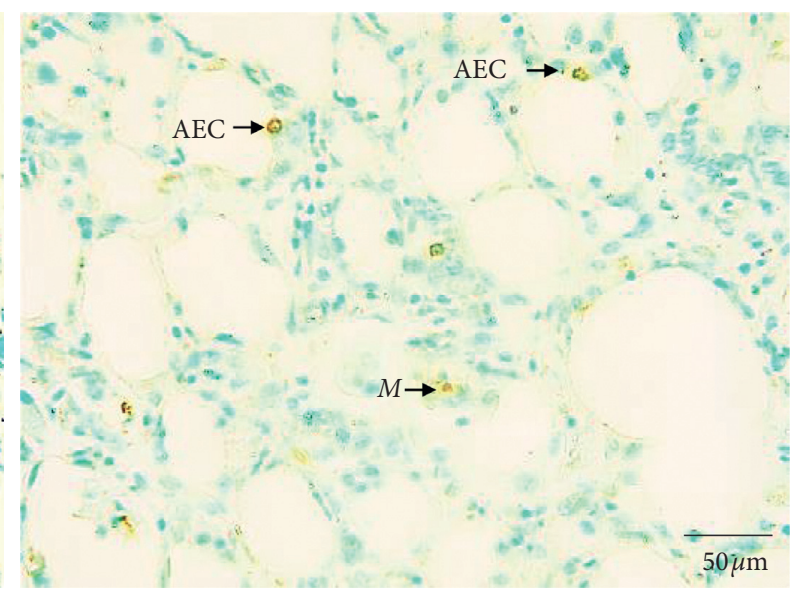

TUNEL +ve (Bleomycin)

(a)

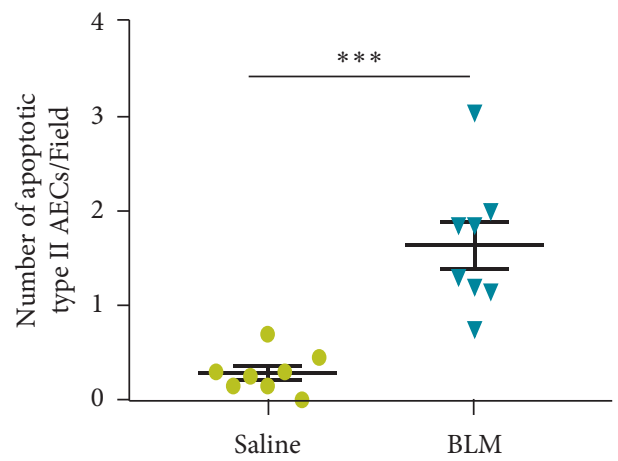

(b)

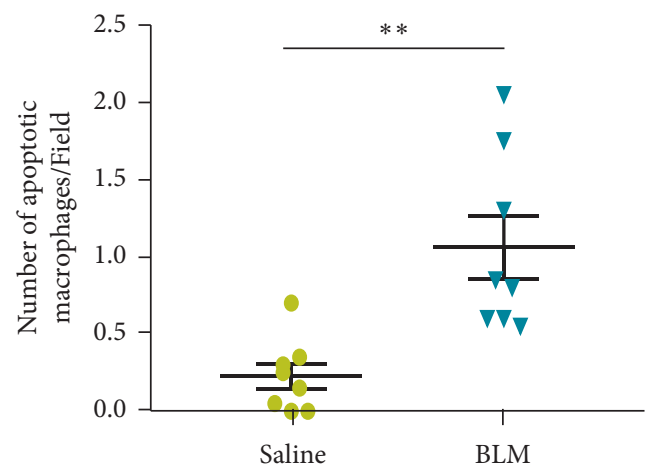

(c)

FIGURE 2: Click-iT ${ }^{\mathrm{TM}}$ TUNEL Colorimetric assay to identify apoptotic type II AECs and macrophages in sheep parenchymal lung tissues. (a) A representative photomicrograph, captured under 40x magnification for the evaluation. Arrows indicate apoptotic type II alveolar epithelial cells (AECs) and macrophages (M). Graphs represent the number of apoptotic type II AECs and macrophages between bleomycin and saline infusion at the fibrotic stage in sheep lung tissues $(n=8)(b, c)$. Each bar represents the mean \pm standard error of the mean. The tissues were sampled from lung segments at post mortem as indicated in Figures $1(\mathrm{a})$ and $1(\mathrm{~b})$. Significance was determined by $t$-test. ${ }^{* *} p<0.001$ and ${ }^{* * *} p<0.0001$.

Colorimetric washing solution. Then, the samples were incubated with Streptavidin-Peroxidase for $30 \mathrm{~min}$ followed by washing with PBS and deionized water. DAB was diluted 1:200 with DAB substrate buffer and added to each sample and kept for 30 seconds to facilitate the reaction. Then, the sections were washed with deionized water to stop the reaction and counter-stained with methyl green. $2 \mathrm{~g}$ of methyl green was dissolved in $100 \mathrm{ml}$ of distilled water and extracted in chloroform to remove the crystal violet. Working solution was prepared by mixing $1: 1$ methyl green with $0.1 \mathrm{M}$ acetate 
buffer. Slides were then counter-stained with methyl green for $10 \mathrm{~min}$, followed by quick dehydration with $70 \%$ and $100 \%$ ethanol and fixed in xylene.

2.6. In Vivo Experimental Design to Evaluate the Therapeutic Effect of Senicapoc and Pirfenidone on the ER Stress and Apoptosis of Type II AEC and Macrophage in Bleomycin-Induced Lung Fibrosis. Sheep lung tissues were obtained from our previous studies $[24,26]$ and a brief description of the experimental design follows. Pulmonary fibrosis was induced in 30 merino sheep $(n=30)$ aged 9-12 months using bleomycin as described above (Figures 3(a) and 3(b)). The sheep were allowed to develop lung fibrosis for another 2 weeks. The sheep were then randomly grouped into three groups $(n=10)$. Each group was then treated with either $30 \mathrm{mg} / \mathrm{kg}$ senicapoc (Icagen Inc., Durham, NC)/pirfenidone (Selleckchem, USA) or methylcellulose (Control) $0.5 \%$ methyl-cell MC (Sigma-Aldrich, Castle Hill, NSW, Australia) orally twice daily. At the end of the treatment regime, sheep were sacrificed by administering intravenous barbiturate (Lethabarb, Virbac Animal Health, Australia) and lung tissues were collected and processed as mentioned above. ER stress and apoptosis of type II AECs and macrophages were evaluated by staining against anti-GRP78 and TUNEL assay, respectively, as mentioned above.

2.7. Quantitative Image Analysis. Images were captured using Leica DM500 microscope. Twenty representative nonoverlapping fields were selected and captured under 40x magnification. Brown stained cells were counted in each field and expressed as means and standard errors of means (mean \pm SEM). Type II AECs and macrophages were identified based on their morphology. Type II AECs are cuboidal cells with centrally located nuclei and the cell is covered by the basement membrane, with a small portion exposed to the alveolar surface [3]. Macrophages were identified by their large irregular shape with a prominent nucleus and granular cytoplasm.

2.8. Statistical Analysis. Statistical analysis was carried out using the software GraphPad Prism version 8.0.1 for Windows (GraphPad Software, La Jolla California USA). Normality testing was performed using D'Agostino-Pearson omnibus normality test. $t$-test was performed to evaluate ER stress and apoptosis in type II AECs and macrophages in sheep lung tissues. The effects of senicapoc and pirfenidone on ER stress and type II AECs and macrophage turnover were evaluated using one-way ANOVA with Tukey's post hoc test to make multiple comparison between groups. An observation with a $p$ value less than $0.05(p<0.05)$ was considered statistically significant.

\section{Results}

3.1. ER Stress Precedes in Bleomycin-Induced Lung Fibrosis in Sheep Model. ER stress was assessed by determining the number of GRP78 immuno-positive cells in sections sampled from differentially treated lung segments collected at post mortem (Figures 1(a) and 1(b)). ER stress in type II AECs and lung macrophages was shown by red cytoplasmic staining, indicating elevated expression of GRP78 proteins in (Figure 1(c)). The rate of ER stress in type II AECs was significantly increased in sheep lung tissues two weeks after they were infused with bleomycin (bleomycin $1.16 \pm 0.17$ vs saline $0.27 \pm 0.06$ cells/field, $p=0.0002$ ) (Figure $1(\mathrm{~d})$ ). Similarly, there was a significant increase in the rate of ER stress in macrophages with the bleomycin infusion when compared to control/saline lung segments (bleomycin $3.30 \pm 0.84$ vs saline $0.46 \pm 0.24$ cells/field, $p=0.006$ ) (Figure $1(\mathrm{e})$ ).

\subsection{Evaluation of Apoptosis in Type II AEC and Macrophages.} Extensive fragmentation of nuclear DNA due to activation of endonucleases is a hallmark of late-stage apoptosis. Fragmented DNA (TUNEL-positive) was indicated by brown nuclear staining in type II AECs and macrophages (Figure 2(a)). As shown in Figure 2(b), the rate of apoptosis in type II AECs increased significantly in bleomycin-infused lung segments when compared to saline-infused lung segments (bleomycin $1.64 \pm 0.25$ vs saline $0.28 \pm 0.07$ apoptotic cells/field, $p=0.0001)$. A significant increase was also observed in the number of apoptotic macrophages in the bleomycin-infused lung segments, when compared to the control lung segments (bleomycin $1.06 \pm 0.20$ vs saline $0.22 \pm 0.08$ apoptotic cells/field, $p=0.0001$ ).

\subsection{KCa3.1 Ion Channel Blockade Significantly Attenuates ER} Stress and Apoptosis in Type II AEC and Lung Macrophages in Lung Fibrosis Induced by Bleomycin. The KCa3.1 ion channel has been shown to play a crucial role in the development of ER stress and apoptosis in type II AECs and lung macrophages [18]. Therefore, we investigated whether ER stress and apoptosis in type II AECs and lung macrophages can be reduced by blocking the $\mathrm{Ca}^{2+}$-activated $\mathrm{K}^{+}$ion channel (KCa3.1) using the KCa3.1-specific ion channel-blocking drug senicapoc. In this experiment, we evaluated the ER stress and apoptosis in lung tissues from the respective groups 7 weeks after the final bleomycin infusion. The group structure and experimental timeline for procedures in this experiment are given in Figures 3(a) and 3(b).

Cytoplasmic expression of the ER stress marker GRP78 in macrophages and AECs is shown for the differentially treated lung segments in Figure 3(c). In control sheep treated with methylcellulose, there were increased numbers of GRP78-positive type II AECs (saline $0.19 \pm 0.04$ vs bleomycin $0.68 \pm 0.12$ cells/field, $n=10, p=0.0003$ ) (Figure $3(\mathrm{~d})$ ) and macrophages (saline $0.32 \pm 0.10$ vs bleomycin $1.46 \pm 0.42$ cells/field, $n=10, p=0.005$ ) (Figure 3(e)) in bleomycininfused lung segments when compared to saline-infused segments. This indicated that the disease model was functioning correctly. When sheep groups treated with either senicapoc or pirfenidone were compared with the methylcellulose control group, the number of AECs expressing the GRP78 protein was significantly reduced in bleomycin-infused lung segments of sheep treated with either senicapoc or pirfenidone (senicapoc $0.26 \pm 0.06$ vs $0.68 \pm 0.12$ cells/field 


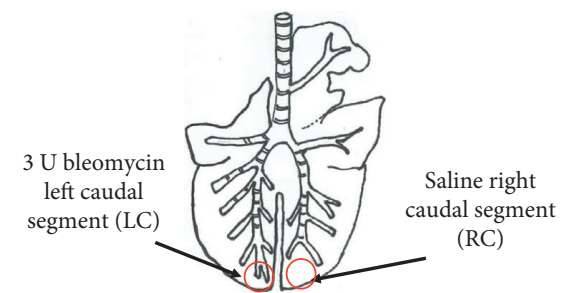

(a)

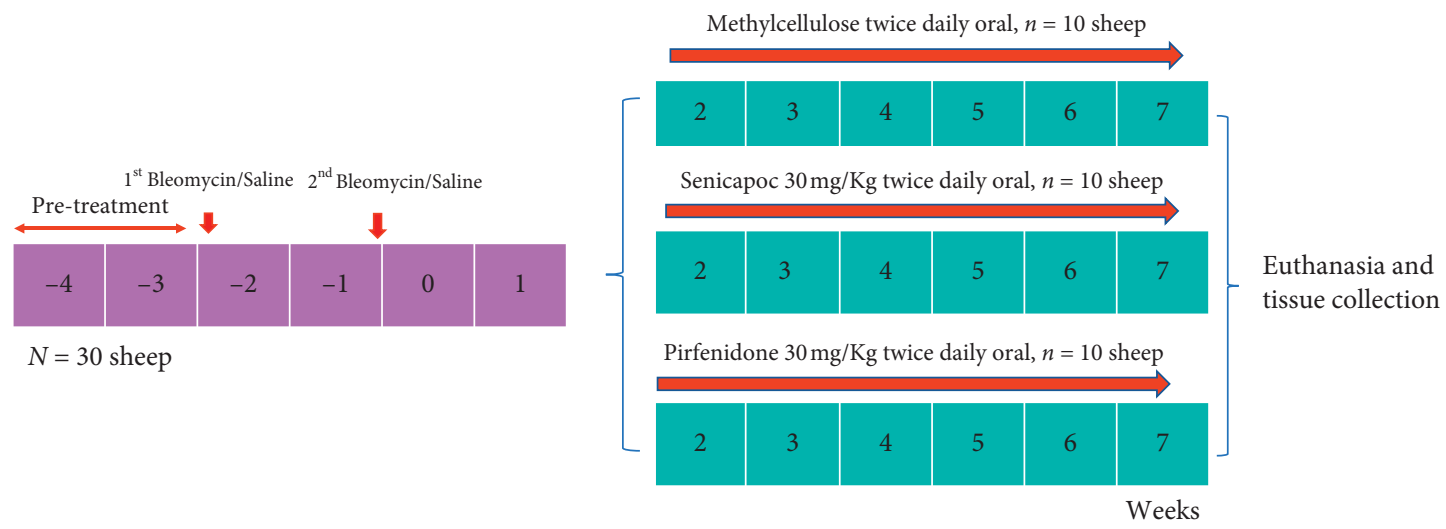

(b)

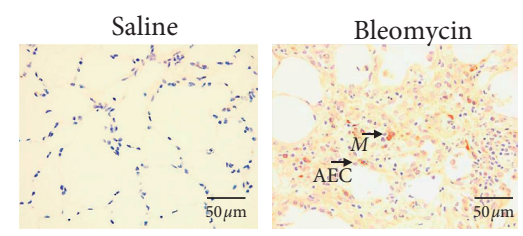

Methylcellulose (GRP78 BiP +ve) Methylcellulose (GRP78 BiP +ve)
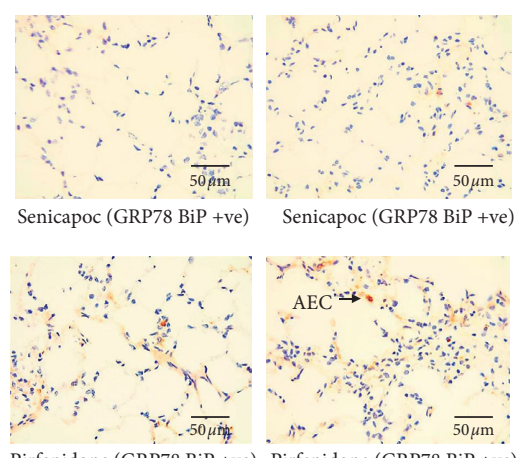

Pirfenidone (GRP78 BiP +ve) Pirfenidone (GRP78 BiP +ve)

(c)

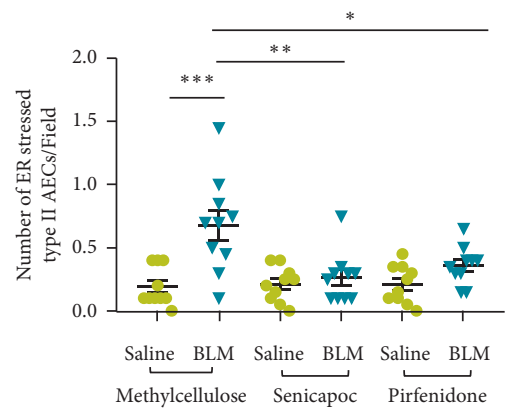

(d)

FIgURE 3: Continued. 


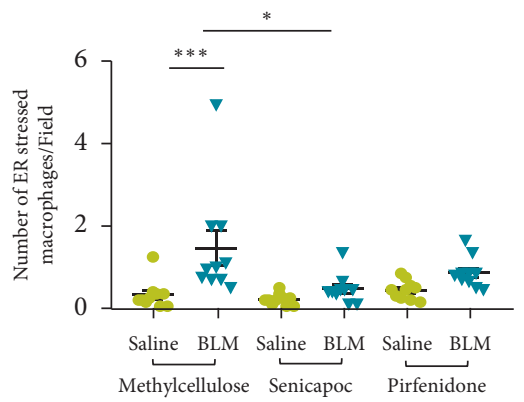

(e)

FIgURE 3: The effects of senicapoc and pirfenidone on ER stress induced by bleomycin in type II AECs and macrophages. (a) In individual sheep, the left caudal lung segment received infusions of bleomycin, while the right caudal lung segment received the saline. (b) Diagram shows the timeline of the bleomycin administration, treatment protocols (methylcellulose (control), senicapoc, pirfenidone) of the three groups of sheep, and the tissue collection. (c) Representative images from immunohistochemical staining against GRP78 to identify ER stress in type II AECs and macrophages in sheep lung segments treated with either methylcellulose (control), senicapoc, or pirfenidone. Arrows indicate ER stressed type II alveolar epithelial cells (AECs) and macrophages (M). (d, e) Graphs show the rate of ER stress in type II AECs and macrophages between the different treatment groups ( $n=10$ sheep/group). The data was collected from twenty representative, non-overlapping fields captured at 40x magnification. Each bar represents the mean \pm standard error of the mean. Significance was determined by one-way ANOVA and a Tukey's post hoc test to make multiple comparisons test between groups. ${ }^{*} p<0.05,{ }^{*}{ }^{*} p<0.001$, and $* * * p<0.0001$.

in vehicle, $n=10 \quad p=0.002$; pirfenidone $0.36 \pm 0.04$ vs $0.68 \pm 0.12$ cells/field in vehicle, $p=0.01$ ) (Figure $3(d)$ ). Furthermore, in senicapoc and pirfenidone-treated sheep, the number of GRP78-positive AECs in bleomycin-infused lung segments was not significantly different to saline-infused lung segments (Figure 3(d)). These data show that the increase in ER stress in AECs due to bleomycin injury was attenuated with treatment with either pirfenidone or senicapoc.

For macrophages, only treatment with senicapoc significantly reduced the number of macrophages in ER stress in lung segments exposed to bleomycin, when compared to vehicle-treated control lung segments injured by bleomycin (senicapoc $0.47 \pm 0.11$ vs $1.46 \pm 0.42$ cells/field in vehicle, $p=0.017$; pirfenidone $0.86 \pm 0.11$ vs $1.46 \pm 0.42$ cells/field in vehicle, $p=0.2$ ) (Figure 3(e)). In senicapoc-treated sheep, the number of GRP78-positive macrophages in bleomycininfused lung segments was not significantly different to saline-infused lung segments (Figure 3(e)). While the number of GRP78-positive macrophages in bleomycin-infused lung segments of pirfenidone-treated sheep was higher than saline-infused lung segments, the data was also not significantly different (Figure 3(e)).

TUNEL-positive fragmented DNA is presented as brown nuclear staining in type II AECs and macrophages for the differentially treated lung segments in Figure 4(a). In control sheep treated with methylcellulose, there were increased numbers of TUNEL-positive type II AECs (saline $0.23 \pm 0.04$ vs bleomycin $0.94 \pm 0.16$ cells/field, $n=10, p<0.0001$ ) (Figure 4(b)) and macrophages (saline $0.14 \pm 0.04$ vs bleomycin $0.53 \pm 0.07$ cells/field, $n=10, p=0.0001$ ) (Figure $4(\mathrm{c})$ ) in bleomycin-infused lung segments when compared to saline-infused segments. This indicated that the disease model was functioning well and the apoptosis in type 2 AECs and macrophages remains high at 7 weeks after the final bleomycin infusion.
When sheep groups treated with either senicapoc or pirfenidone were compared with the methylcellulose control group, the number of TUNEL-positive AECs was significantly reduced in bleomycin-infused lung segments of sheep treated with senicapoc (senicapoc $0.43 \pm 0.06$ vs $0.94 \pm 0.16$ cells/field in vehicle, $n=10, p=0.003$ ) (Figure 4(b)). While the number of apoptotic AECs was lower with pirfenidone treatment, when compared with methylcellulose control treatment, the difference was not significant (pirfenidone $0.65 \pm 0.07$ vs $0.94 \pm 0.16$ cells/field in vehicle, $n=10, p=0.2$ ) (Figure 4(b)). In both senicapoc- and pirfenidone-treated sheep, the number of TUNEL-positive AECs in bleomycininfused lung segments was not significantly different to saline-infused lung segments (Figure 4(b)).

For macrophages, treatment with senicapoc significantly reduced the number of apoptotic of macrophages in lung segments exposed to bleomycin, when compared to vehicletreated control lung segments injured by bleomycin (senicapoc $0.31 \pm 0.04$ vs $0.53 \pm 0.07$ cells/field in vehicle, $n=10$, $p=0.04$; pirfenidone $0.35 \pm 0.04$ vs $0.53 \pm 0.07$ cells/field in vehicle, $n=10, p=0.1$ ) (Figure $4(\mathrm{c})$ ).

\section{Discussion}

This study is the first to report that the bleomycin treatment increases ER stress and apoptosis in type II AECs and lung macrophages, and this effect is significantly ameliorated by in vivo blockade of the KCa3.1 ion channel with the drug senicapoc. Our previous studies using the sheep pulmonary fibrosis model reported that there were significant reductions in lung fibrosis and microvascular remodeling in sheep treated with senicapoc $[23,24]$. The attenuated lung pathology in senicapoc-treated sheep coincided with improved lung function in these animals [24]. Findings of the current study show that blockade of the KCa3.1 ion channel reduces ER stress and apoptosis in type II AECs and macrophages, 


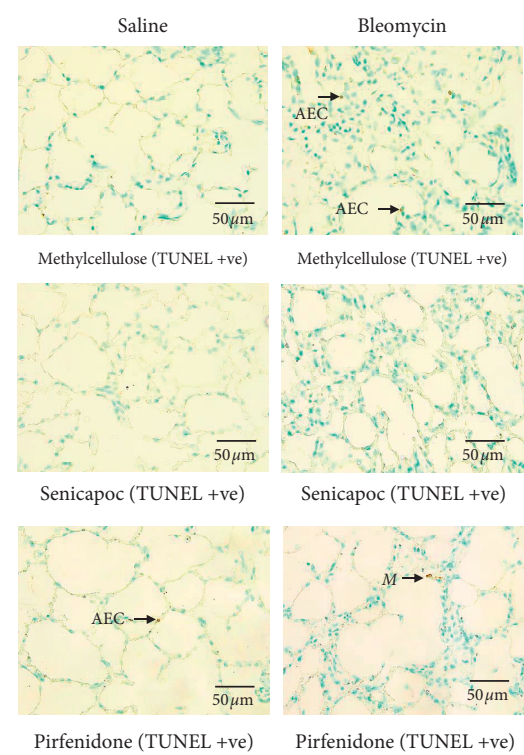

(a)

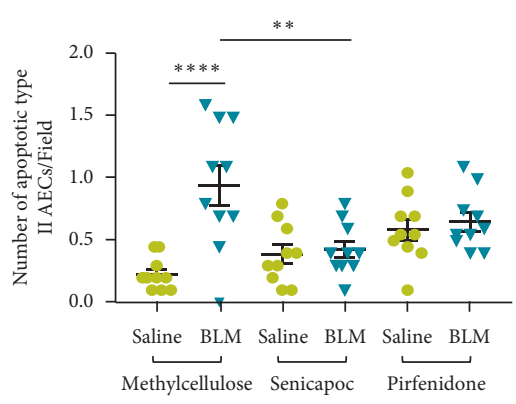

(b)

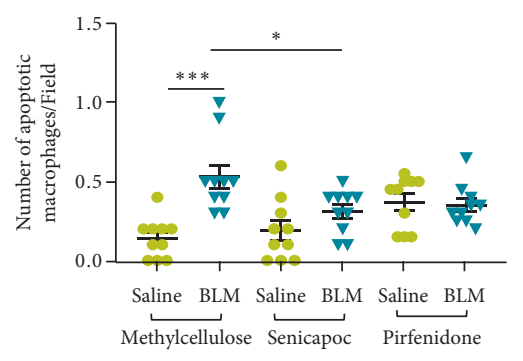

(c)

Figure 4: Effects of senicapoc and pirfenidone treatment on apoptosis induced by bleomycin in type II AECs and macrophages. (a) Representative images from colorimetric TUNEL assays to identify apoptotic type II AECs and macrophages in sheep lung segments treated with either methylcellulose (control), senicapoc, or pirfenidone. Arrows indicate apoptotic type II alveolar epithelial cells (AECs) and macrophages (M). (b, c) Graphs represent the rate of apoptosis in type II AECs and macrophages between the different treatment groups ( $n=10$ sheep/group). The data was collected from twenty representative, non-overlapping fields captured at 40x magnification. Each bar represents the mean \pm standard error of the mean. The group structure and experimental timeline for procedures in this experiment are given in Figures 3(a) and 3(b). Significance was determined by one-way ANOVA and a Tukey's post hoc test to make multiple comparisons test between groups. ${ }^{*} p<0.05,{ }^{* *} p<0.001$, and ${ }^{* * *} p<0.0001$.

and this effect presumably contributes to the reduced pathology and improved lung function observed in this sheep model of pulmonary fibrosis.

Chronic ER stress-mediated apoptosis in type II AECs and macrophages has been identified as one of the principle pathogenic mechanisms in IPF $[12,27]$. But the underlying mechanisms of ER stress-mediated apoptosis are complex and not yet fully understood [28]. Our results show that the prevalence of ER stress and apoptosis in type II AEC and macrophages was high 2 weeks after the final bleomycin infusion and remained at significantly high levels for at least 7 weeks after bleomycin injury.

Caution should be noted that these conclusions are based on relatively small numbers of apoptotic and ER stressed cells in the differentially treated lung samples. The range of ER stress-, or apoptotic-positive cells, observed in our study, was within 0-6 cells per field. Although statistically significant reductions in these indices were found for the drug treatments, the question that needs to be asked is whether these observed indices reductions have clinically relevant outcomes. Some of the low positive cell counts per field are due to the low overall density of cells in lung parenchyma, compared to other organs, resulting from the relatively high air-to-tissue-space ratio in the gas exchange region of the lung. In a previous study of lung idiopathic interstitial pneumonias, the frequency of TUNEL-positive cells in lung epithelium was a relatively low one to three percent of total lung epithelial cells counted compared to cells in culture [29]. The authors argue that apoptotic cells are rapidly phagocytosed and thus are only present for a relatively short time under light, or electron, microscopy. Thus, while the frequency of TUNEL-positive apoptotic cells visualized in tissue specimens is low, this small number may still reflect a considerable magnitude of cell loss [30]. Overall, the reductions in cellular apoptosis and ER stress observed with pirfenidone and senicapoc in the current sheep study should be seen in context with our previous clinical findings with these drugs. We have previously shown significant improvements in lung compliance, fibrotic scores, vascular remodeling, and collagen deposition in sheep treated with the KCa3.1 channel blocker senicapoc and the FDA-approved drug pirfenidone $[23,24,26]$. In the current study, the reduction of apoptosis and ER stress after treatment with these drugs is part of their overall clinical effectiveness.

The relationship between these intracellular events and the KCa3.1 ion channel needs to be further elucidated. We have reported that the KCa3.1 ion channel is widely expressed in alveolar epithelial cells [24]. Membrane potential and $\mathrm{Ca}^{2+}$ signaling of alveolar epithelial cells are mainly regulated by $\mathrm{KCa} 3.1$ channels in order to maintain critical cellular functions such as activation, migration, and proliferation $[18,31]$. The KCa3.1 ion channel is a voltageindependent $\mathrm{K}^{+}$channel that opens when intracellular $\mathrm{Ca}^{2+}$ levels increase, resulting in $\mathrm{Ca}^{2+}$-activated $\mathrm{K}^{+}$efflux. This process plays an important role in regulation of cellular volume. When there is a continuous stimulus, the KCa3.1 ion channels become activated $[18,32]$. This will result in substantial loss of intracellular $\mathrm{K}^{+}$ions to the extracellular 
compartment, and more than $50 \%$ of the intracellular $\mathrm{K}^{+}$can be lost via efflux [18]. Depletion of intracellular $\mathrm{K}^{+}$ions induces apoptosis via caspase activation, cytochrome $\mathrm{c}$ release, and DNA degradation [33-35]. It is likely that the in vivo blockade of $\mathrm{KCa} 3.1$ channels in the current study maintains ion balance between the intracellular and extracellular compartments, which in turn leads to a reduction of apoptosis [36].

The accumulation of unfolded proteins, imbalance of $\mathrm{Ca}^{2+}$ homeostasis, oxidative stress, and ischemia are some of the factors responsible for inducing ER stress in degenerative and fibrotic disorders in multiple organs [14, 37]. GRP78 is an ER stress chaperone protein that plays an important role in maintaining ER protein synthesis, folding, and intracellular calcium homeostasis $[10,38]$. By evaluating the increased expression levels of GRP78 protein in type II AECs and macrophages, we demonstrated that the rate of ER stress in both type II AECs and macrophages was significantly retarded by blocking the KCa3.1 ion channel with senicapoc. Our findings are consistent with previous work evaluating the correlation between the calcium-dependent ER stress and KCa3.1 channels, where blockade or genetic deletion of the KCa3.1 ion channel was shown to reduce $\mathrm{Ca}^{2+}$ overload and attenuate ER stress in astrocytes of both humans and mice [39]. While these experiments were dealing with human and mouse brain cells, the findings are consistent with the reduction of bleomycin-induced ER stress in type II AECs and lung macrophages we observed in sheep treated with senicapoc.

We found that administration of the approved anti-fibrotic drug pirfenidone also reduced the rate of apoptosis in type II AECs and macrophages in bleomycin-infused segments. The underlying molecular mechanisms responsible for pirfenidone anti-fibrotic action are not fully understood. It is known that pirfenidone inhibits TGF- $\beta$ stimulated collagen synthesis, myofibroblast differentiation, and fibrogenic activity of human lung fibroblasts [4-6]. Alveolar macrophages, type II AECs, myofibroblasts, and eosinophils are the main sources of TGF- $\beta$ in lung fibrosis [40]. As TGF$\beta$ is a potent inducer of apoptosis of type II AECs, pirfenidone may exert its effect through suppression of the TGF$\beta$ pathway.

\section{Conclusion}

In vivo blockade with a specific inhibitor of the Kca3.1 ion channel alleviates the ER stress and apoptosis in type II AECs and macrophages. Our data provides an insight into how this drug interacts with these cell types in fibrotic lung tissues and thus contributes to the understanding of its antifibrotic effects in pulmonary fibrosis.

\section{Abbreviations}

IPF: Idiopathic pulmonary fibrosis

ER: $\quad$ Endoplasmic reticulum

AECs: Alveolar epithelial cells

KCa3.1: Calcium-activated potassium ion channel

GRP78: Glucose regulated protein 78
FDA: $\quad$ Food and drug administration

TGF- $\beta$ : $\quad$ Transforming growth factor $\beta$

OCT: Optimal cutting temperature

PBS: $\quad$ Phosphate buffer saline

TdT: Terminal Deoxynucleotidyl Transferase

BSA: Bovine serum albumin

NSS: Normal sheep serum

TUNEL: Terminal Deoxynucleotidyl Transferase-dUTP nick end labeling

DAB: Diaminobenzidine

SEM: $\quad$ Standard error of means

LC: $\quad$ Left caudal

RC: $\quad$ Right caudal

BLM: Bleomycin.

\section{Data Availability}

The raw datasets in this study can be obtained from the corresponding author upon request.

\section{Ethical Approval}

All experimental procedures relating to sheep experiments and sample collection were approved by Animal Experimentation Ethics Committee, University of Melbourne (Parkville, VIC, Australia), and adhere to the Australian Code of Conduct for the Care and Use of Animals for Scientific Purposes.

\section{Conflicts of Interest}

The authors declare that they have no conflicts of interest.

\section{Authors' Contributions}

UEP was the principal researcher for the study; she conducted the laboratory work statistical analyses and interpretation. UEP and KJS participated in the concept and study design and prepared the manuscript. LO and SNVD advised on study methodology. AS assisted in immunohistochemistry. AS and HBD assisted with the preparation of the manuscript. All authors read and approved the final manuscript.

\section{Acknowledgments}

The authors would like to thank Mr. Paul Benham, Technician, Anatomic Pathology, University of Melbourne, for the assistance in immunohistochemistry for sheep.

\section{References}

[1] N. Sakai and A. M. Tager, "Fibrosis of two: epithelial cellfibroblast interactions in pulmonary fibrosis," Biochimica et Biophysica Acta (BBA)-Molecular Basis of Disease, vol. 1832, no. 7, pp. 911-921, 2013.

[2] G. Raghu, M Remy-Jardin, J. L Myers et al., "Diagnosis of idiopathic pulmonary fibrosis. An official ATS/ERS/JRS/ ALAT clinical practice guideline," American Journal of 
Respiratory and Critical Care Medicine, vol. 198, no. 5, pp. e44-e68, 2018.

[3] M. Plataki, A. V. Koutsopoulos, K. Darivianaki, G. Delides, N. M. Siafakas, and D. Bouros, "Expression of apoptotic and antiapoptotic markers in epithelial cells in idiopathic pulmonary fibrosis," Chest, vol. 127, no. 1, pp. 266-274, 2005.

[4] K. Meyer and C. Decker, "Role of pirfenidone in the management of pulmonary fibrosis," Therapeutics and Clinical Risk Management, vol. 13, pp. 427-437, 2017.

[5] G. Margaritopoulos, E. Vasarmidi, and K. Antoniou, "Pirfenidone in the treatment of idiopathic pulmonary fibrosis: an evidence-based review of its place in therapy," Core Evidence, vol. 11, pp. 11-22, 2016.

[6] U. Costabel, E. Bendstrup, V. Cottin et al., "Pirfenidone in idiopathic pulmonary fibrosis: expert panel discussion on the management of drug-related adverse events," Advances in Therapy, vol. 31, no. 4, pp. 375-391, 2014.

[7] S.-J. Kim, P. Cheresh, R. Jablonski, D. Williams, and D. Kamp, "The role of mitochondrial DNA in mediating alveolar epithelial cell apoptosis and pulmonary fibrosis," International Journal of Molecular Sciences, vol. 16, no. 9, pp. 21486-21519, 2015.

[8] S. Johnson, S. B. Shaikh, F. Muneesa, B. Rashmi, and Y. P. Bhandary, "Radiation induced apoptosis and pulmonary fibrosis: curcumin an effective intervention?" International Journal of Radiation Biology, vol. 96, no. 6, pp. 709-717, 2020.

[9] A. J. Byrne, T. M. Maher, and C. M. Lloyd, "Pulmonary macrophages: a new therapeutic pathway in fibrosing lung disease?" Trends in Molecular Medicine, vol. 22, no. 4, pp. 303-316, 2016.

[10] Y. Li, "Effects of calcium Ionophore A23187 on the apoptosis of hepatic stellate cells stimulated by transforming growth factor-beta1," Cell Stress and Chaperones, vol. 23, p. 1, 2018.

[11] V. Sharma, R. Kaur, A. Bhatnagar, and J. Kaur, "Low-pHinduced apoptosis: role of endoplasmic reticulum stress-induced calcium permeability and mitochondria-dependent signaling," Cell Stress and Chaperones, vol. 20, no. 3, pp. 431-440, 2015.

[12] W. E. Lawson, P. F. Crossno, V. V. Polosukhin et al., "Endoplasmic reticulum stress in alveolar epithelial cells is prominent in IPF: association with altered surfactant protein processing and herpesvirus infection," American Journal of Physiology-Lung Cellular and Molecular Physiology, vol. 294, no. 6, pp. L1119-L1126, 2008.

[13] A. J. Ryan, J. L. Larson-Casey, C. He, S. Murthy, and A. B. Carter, "Asbestos-induced disruption of calcium homeostasis induces endoplasmic reticulum stress in macrophages," Journal of Biological Chemistry, vol. 289, no. 48, pp. 33391-33403, 2014.

[14] J. A. Kropski and T. S. Blackwell, "Endoplasmic reticulum stress in the pathogenesis of fibrotic disease," Journal of Clinical Investigation, vol. 128, no. 1, pp. 64-73, 2018.

[15] M. Thamsen, "Small molecule inhibition of IRE1alpha kinase/ RNase has anti-fibrotic effects in the lung," PLoS ONE, vol. 14, no. 1, Article ID e0209824, 2019.

[16] W. A. Wuyts, C. Agostini, K. M. Antoniou et al., "The pathogenesis of pulmonary fibrosis: a moving target," European Respiratory Journal, vol. 41, no. 5, pp. 1207-1218, 2013.

[17] L. Zhang, Y. Wang, N. S. Pandupuspitasari et al., "Endoplasmic reticulum stress, a new wrestler, in the pathogenesis of idiopathic pulmonary fibrosis," American Journal of Translational Research, vol. 9, no. 2, pp. 722-735, 2017.

[18] H. Wulff and N. A. Castle, "Therapeutic potential of KCa3.1 blockers: recent advances and promising trends," Expert
Review of Clinical Pharmacology, vol. 3, no. 3, pp. 385-396, 2010.

[19] L. Sevelsted Moller, "The calcium-activated potassium channel KCa3.1 is an important modulator of hepatic injury," Science Reports, vol. 6, p. 28770, 2016.

[20] A. Olivan-Viguera, "Inhibition of intermediate-conductance calcium-activated $\mathrm{K}$ channel (KCa3.1) and fibroblast mitogenesis by alpha-linolenic acid and alterations of channel expression in the lysosomal storage disorders, fabry disease, and niemann pick C," Frontiers in Physiology, vol. 8, p. 39, 2017.

[21] I. Grgic, E. Kiss, B. P. Kaistha et al., "Renal fibrosis is attenuated by targeted disruption of KCa3.1 potassium channels," Proceedings of the National Academy of Sciences, vol. 106, no. 34, pp. 14518-14523, 2009.

[22] K. M. Roach, "The $\mathrm{K}+$ channel KCa3.1 as a novel target for idiopathic pulmonary fibrosis," PLoS ONE, vol. 8, no. 12, Article ID e85244, 2013.

[23] H. B. Derseh, "KCa3.1 channel blockade attenuates microvascular remodelling in a large animal model of bleomycininduced pulmonary fibrosis," Science Reports, vol. 9, no. 1, p. 19893, 2019.

[24] L. Organ, B. Bacci, E. Koumoundouros et al., "Inhibition of the KCa3.1 channel alleviates established pulmonary fibrosis in a large animal model," American Journal of Respiratory Cell and Molecular Biology, vol. 56, no. 4, pp. 539-550, 2017.

[25] L. Organ, B. Bacci, E. Koumoundouros et al., “A novel segmental challenge model for bleomycin-induced pulmonary fibrosis in sheep," Experimental Lung Research, vol. 41, no. 3, pp. 115-134, 2015.

[26] S. N. V. Dewage, "The efficacy of pirfenidone in a sheep model of pulmonary fibrosis," American Journal of Respiratory and Critical Care Medicine, vol. 45, no. 9-10, pp. 310-322, 2019.

[27] M. Korfei, C. Ruppert, P. Mahavadi et al., "Epithelial endoplasmic reticulum stress and apoptosis in sporadic idiopathic pulmonary fibrosis," American Journal of Respiratory and Critical Care Medicine, vol. 178, no. 8, pp. 838-846, 2008.

[28] R. V. Rao, H. M. Ellerby, and D. E. Bredesen, "Coupling endoplasmic reticulum stress to the cell death program," Cell Death \& Differentiation, vol. 11, no. 4, pp. 372-380, 2004.

[29] K. Kuwano, N. Hagimoto, T. Maeyama et al., "Mitochondriamediated apoptosis of lung epithelial cells in idiopathic interstitial pneumonias," Laboratory Investigation, vol. 82, no. 12, pp. 1695-1706, 2002.

[30] J. M. Isner, M. Kearney, S. Bortman, and J. Passeri, "Apoptosis in human atherosclerosis and restenosis," Circulation, vol. 91, no. 11, pp. 2703-2711, 1995.

[31] B. M. Brown, B. Pressley, and H. Wulff, "KCa3.1 channel modulators as potential therapeutic compounds for glioblastoma," Current Neuropharmacology, vol. 16, no. 5, pp. 618-626, 2018.

[32] T. L. Peña, S. H. Chen, S. F. Konieczny, and S. G. Rane, "Ras/ MEK/ERK up-regulation of the fibroblast KCaChannel FIK is a common mechanism for basic fibroblast growth factor and transforming growth factor- $\beta$ suppression of myogenesis," Journal of Biological Chemistry, vol. 275, no. 18, pp. 1367713682, 2000.

[33] M. Song and S. P. Yu, "Ionic regulation of cell volume changes and cell death after ischemic stroke," Translational Stroke Research, vol. 5, no. 1, pp. 17-27, 2014.

[34] M. I. Panayiotidis, C. D. Bortner, and J. A. Cidlowski, "On the mechanism of ionic regulation of apoptosis: would the $\mathrm{Na}+$ / K+-ATPase please stand up?" Acta Physiologica (Oxf), vol. 187, no. 1-2, pp. 205-215, 2006. 
[35] A. Kondratskyi, K. Kondratska, R. Skryma, and N. Prevarskaya, "Ion channels in the regulation of apoptosis," Biochimica et Biophysica Acta (BBA)-Biomembranes, vol. 1848, no. 10, pp. 2532-2546, 2015.

[36] P. A. Lang, "Role of Ca2+-activated $\mathrm{K}+$ channels in human erythrocyte apoptosis," EXCLI Journal, vol. 285, pp. 15531560, 2003.

[37] N. Khodaee, "Endoplasmic reticulum membrane potassium channel dysfunction in high fat diet induced stress in rat hepatocytes," EXCLI Journal, vol. 13, pp. 1075-1087, 2014.

[38] M. Liu, G. Shi, A. Zhou, C. E. Rupert, K. L. K. Coulombe, and S. C. Dudley, "Activation of the unfolded protein response downregulates cardiac ion channels in human induced pluripotent stem cell-derived cardiomyocytes," Journal of Molecular and Cellular Cardiology, vol. 117, pp. 62-71, 2018.

[39] $\mathrm{Z} . \mathrm{Yu}$, " $\mathrm{Ca}(2+)$-dependent endoplasmic reticulum stress correlation with astrogliosis involves upregulation of KCa3.1 and inhibition of AKT/mTOR signaling," Journal of Neuroinflammation, vol. 15, no. 1, p. 316, 2018.

[40] X. Yue, B. Shan, and J. A. Lasky, "TGF-beta: titan of lung fibrogenesis," Journal of Neuroinflammation, vol. 6, no. 2, 2010 . 


\section{University Library}

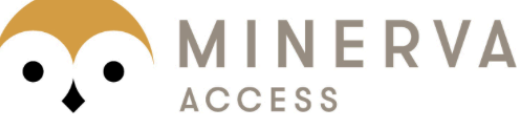

A gateway to Melbourne's research publications

Minerva Access is the Institutional Repository of The University of Melbourne

Author/s:

Perera, UE;Organ, L;Dewage, SNV;Derseh, HB;Stent, A;Snibson, KJ

Title:

Increased Levels of ER Stress and Apoptosis in a Sheep Model for Pulmonary Fibrosis Are Alleviated by In Vivo Blockade of the KCa3.1 Ion Channel

Date:

2021-03-20

\section{Citation:}

Perera, U. E., Organ, L., Dewage, S. N. V., Derseh, H. B., Stent, A. \& Snibson, K. J. (2021). Increased Levels of ER Stress and Apoptosis in a Sheep Model for Pulmonary Fibrosis Are Alleviated by In Vivo Blockade of the KCa3.1 Ion Channel. CANADIAN RESPIRATORY JOURNAL, 2021, https://doi.org/10.1155/2021/6683195.

Persistent Link:

http://hdl.handle.net/11343/280401

License:

CC BY 